\title{
Trigeminal Mononeuropathy: First Clinical Manifestation of Breast Cancer
}

\author{
Germán Morís $^{a}$ Mar Pérez-Peña ${ }^{b}$ Eva Miranda ${ }^{c}$ José López Anglada ${ }^{c}$ Renee Ribacoba $^{a}$ \\ Carmen González ${ }^{a}$
}

Services of a Neurology, ${ }^{\mathrm{b}}$ Radiology and ${ }^{\mathrm{c}}$ Pathology, Hospital Alvarez-Buylla, Mieres, Spain

\section{Dear Sir,}

Trigeminal neuropathy is a well-recognized manifestation of neoplastic lesions. Tumors arising from the cerebellopontine angle or invasive primary tumors involving the neck and nasopharynx may cause trigeminal nerve dysfunction concomitantly with other cranial nerve palsies [1]. Metastatic trigeminal mononeuropathy is caused by a metastasis to the leptomeninges, dura mater or cavernous sinus [2-5]. The occurrence of isolated metastasis to the trigeminal ganglion from a distant, solid tumor is rare and it develops as an expression of known cancer [6-8]. We report a patient with a painful trigeminal mononeuropathy caused by a breast cancer metastasis as the first clinical manifestation of her cancer.

\section{Case Report}

A previously healthy 45 -year-old woman presented with a 2-month history of numbness of the left side of her face. Neurological examination disclosed hypaesthesia in all three divisions of the left trigeminal nerve, as well as unilateral atrophy of the temporal and masseter muscles. The ipsilateral corneal reflex was abolished. There were no other cranial nerve abnormalities, and no other abnormal central nervous system signs were elicited. Magnetic resonance imaging of the brain was performed, showing an enlargement of the left trigeminal nerve mass and gasserian ganglion. The mass enhanced homogeneously after gadolinium injection (fig. 1). There were no brain metastases or abnormality of the me- ninges. Analyses of the cerebrospinal fluid were normal including cytology. Multiple lytic lesions of the cranium were also found. A computerized tomography (CT) scan confirmed the erosion of the left petrous apex as well as multiple bone lesions. Full examination of the patient's blood yielded normal results. Mammography showed only fibrocystic breast disease. A CT scan did not reveal metastases of malignancy into the abdomen or chest. Pelvis bone marrow biopsy revealed carcinoma cells that were positive for oestrogen and progesterone receptors (fig. 2). These findings were consistent with metastatic breast disease.

\section{Discussion}

Tumoral disease could be the underlying condition in trigeminal neuropathy. According to the report of a study of 9 patients with trigeminal sensory neuropathy of nontraumatic cause, with trigeminal dysfunction resulting from malignant disease only in 4 of them, 3 patients had metastatic involvement of the jaw (prostatic carcinoma, breast carcinoma and pulmonary adenocarcinoma) and 1 had a cerebellar glioblastoma [9].

The numb chin syndrome, also known as mental nerve neuropathy, is characterized by numbness confined to the chin and lower lip. Second to dental causes are malignant neoplasms. The mechanism of such a manifestation can be a direct compression of the mental, the inferior alveolar or the mandibular nerves by metastases to the

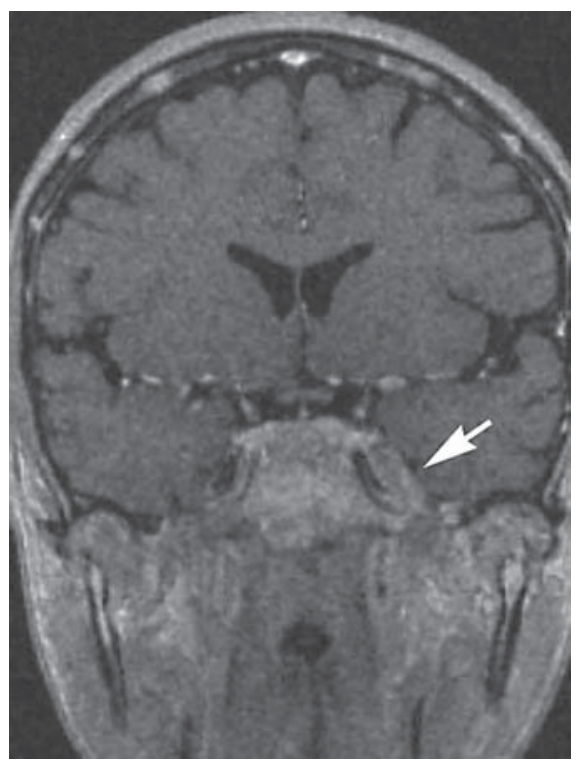

Fig. 1. Coronal contrast-enhanced $T_{1}$ weighted magnetic resonance image showing enhancement and thickening of the left trigeminal ganglion and proximal third division of the trigeminal nerve (arrow). Multiple skull metastases are also evident.

mandible, intracranial metastases, leptomeningeal seeding and neoplastic perineural infiltration. The most frequent histological cancer subtypes reported are lymphomas and breast cancer [5].

The base of the skull could be involved by cancer disease. Greenberg et al. [4] defined 5 clinical syndromes of metastases to

\section{KARGER}

Fax +4161306 1234 E-Mail karger@karger.ch www.karger.com
Dr. G. Morís

Servicio de Neurología, Hospital San Agustín

C/Camino de Heros 4

ES-33400 Avilés, Asturias (Spain)

Tel. +34 985123 000, Fax +34 985123 010, E-Mail gmorist@seneurologia.org 


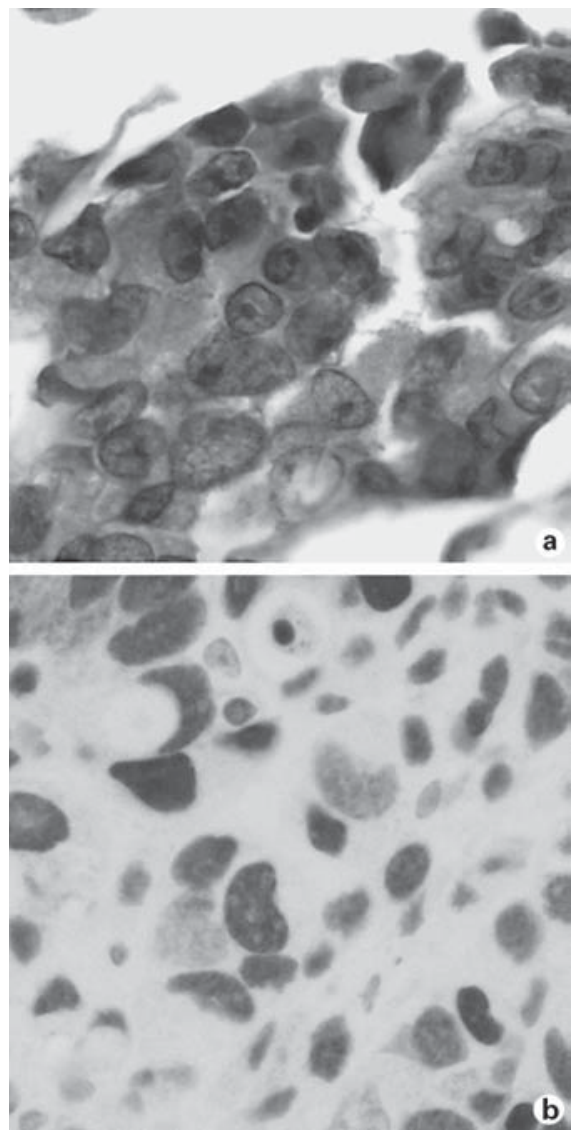

Fig. 2. a Light photomicrographs of the biopsy specimen demonstrating large groups of malignant cells with lack of cellular cohesion. Haematoxylin-eosin staining. $\times 40$. b Photomicrographs of tumour sections with immunohistochemical staining, the tumour cells are positive for oestrogen receptors. Original magnification $\times 40$. the base skull; one of them is the middle fossa (gasserian ganglion) syndrome which is characterized by facial numbness but other cranial nerves are likely to be involved. They described 7 patients with breast metastases in the middle fossa, involving either the gasserian ganglion or branches of the trigeminal nerve, but the interval from diagnosis of the primary breast cancer was from 2 months to 16 years (median 66 months). Moreover, approximately $30 \%$ of women with a diagnosis of breast cancer will develop bone metastases; among patients with metastatic disease, $70 \%$ will have skeletal involvement [10]. Finally, brain metastases from breast cancer occur on average 42-60 months after the diagnosis of the primary lesion [6].

In the present case, there was no evidence of extraskeletal involvement, and bone disease was asymptomatic. The first symptoms of the malignant breast disease was facial hypaesthesia. This patient did not have a numb chin syndrome according to the involvement of the tree branches of the trigeminal nerve including its motor branch. Neuroradiological examination revealed infiltration of the trigeminal ganglion. Initially, the bone of the middle fossa was invaded by haematogenous dissemination from the extracranial malignancy and secondarily involved the trigeminal ganglion by direct extension from the temporal bone. Finally, malignant cells had a neural extension along the trigeminal nerve, especially the third branch. In conclusion, we would like to emphasize that dysfunction of the trigeminal nerve could be the first and unique sign of a remote malignant disease.

\section{References}

1 Beck DW, Menezes AH: Lesions in Meckel's cave: variable presentation and pathology. $\mathrm{J}$ Neurosurg 1987;67:684-689.

2 Schabet M, Kloeter I, Adam T, Heidemann E, Wietholter $\mathrm{H}$ : Diagnosis and treatment of meningeal carcinomatosis in ten patients with breast cancer. Eur Neurol 1986;25:403-411.

3 Dethy S, Piccart MJ, Paesmans M, van Houtte P, Klastersky J: History of brain and epidural metastases from breast cancer in relation with the disease evolution outside the central nervous system. Eur Neurol 1995;35:38-42.

4 Greenberg HS, Deck MD, Vikram B, Chu FC, Posner JB: Metastasis to the base of the skull: clinical findings in 43 patients. Neurology 1981;31:530-537.

5 Massey EW, Moore J, Schold SC: Mental neuropathy from systemic cancer. Neurology 1981;31:1277-1281.

6 Hirota N, Fujimoto T, Takahashi M, Fukushima Y: Isolated trigeminal nerve metastases from breast cancer: an unusual cause of trigeminal mononeuropathy. Surg Neurol 1998;49: 558-561.

7 Iñiguez C, Mauri JA, Larrode P, Perez J, Pina MA, Morales F: Mandibular neuropathy due to infiltration of the Gasser ganglion. Rev Neurol 1997;25:1092-1094.

8 Nakano I, Iwasaki K, Kondo A: Solitary metastatic breast carcinoma in a trigeminal nerve mimicking a trigeminal neurinoma: case report. J Neurosurg 1996;85:677-680.

9 Shotts RH, Porter SR, Kumar N, Scully C: Longstanding trigeminal sensory neuropathy of nontraumatic cause. Oral Surg Oral Med Oral Pathol Oral Radiol Endod 1999;87:572576.

10 Lipton A: Bisphosphonates and metastatic breast carcinoma. Cancer 2003;97:S848S853. 